\title{
Early factors at infancy that predict a developing neurobehavioral disorder - A pilot study
}

\author{
Gurevitz M* \\ Maccabi Health Services, Hasharon region, Israel
}

\begin{abstract}
Background: The increasing incidence of Neurobehavioral Disorders (NBDs), such as Autism Spectrum Disorder (ASD), Attention Deficit Hyperactivity Disorder (ADHD) and Developmental Coordination Disorder (DCD), is of major concern in public health. Although early detection of a developing NBD is critical to enable intervention while brain plasticity is prominent, no screening tool for NBD development at infancy is available. The aim here was to assess whether risk factors that predict forthcoming NBDs can be identified at early infancy.

Methods: The Israeli Health Organization provides a developmental follow-up system since birth at special well-baby-care clinics ('Tipat Chalav'). The documented data was used to examine the charts of 161 babies, who later in life (2-10 years of age) were diagnosed for NBDs (47 ASD, 56 DCD, 58 ADHD). Their medical history at 0-18 months of age was compared to that of 58 babies with typical development (control group). Ten covariates including 28 parameters were collected, compared and statistically analysed.

Results: This analysis indicated that deviation from trajectories of seven parameters (gestational age, birth weight, head circumference percentile, weight percentile, gross motor development, difficulties in speech and communication) may collectively predict the development of ASD with 85\% probability. Deviation from trajectories of the above first five parameters may collectively predict the development of DCD with $72 \%$ probability. Early risk factors that may predict ADHD development with $58 \%$ probability were previously described (Gurevitz et al 2014).

Conclusion: This cohort retrospective study illuminates risk factors at infancy that may predict the development of NBDs. Most prominent are deviations from weight followed by deviations in head circumference trajectories, and delay in motor development due to muscle strength and tone irregularities. Identification of risk factors at infancy is crucial for early intervention programs, such as prevention of rapid changes in weight and manipulations to strengthen motor development and the corresponding neural circuits. The sooner risk factors are recognized, more efficient an early tailored bio-psycho-social treatment might be.
\end{abstract}

Trial registration: Retrospectively registered.

\begin{abstract}
Abbreviations: ADHD: Attention Deficit Hyperactivity Disorder; ASD: Autistic Spectrum Disorder; BW: Birth Weight; CS: Cesarean Section; DP: Deformational Plagiocephaly; DCD: Developmental Coordination Disorder; GA: Gestational Age; GM: Gross Motor; HCp: Head Circumference percentile; Hp: Height percentile; LD: Learning Disorders; MD: Mental Disorders; NBD: Neurobehavioral Disorder; Wp: Weight percentile.
\end{abstract}

\section{Background}

Neurobehavioral Disorders (NBDs), such as Autism Spectrum Disorder (ASD), Attention Deficit Hyperactivity Disorder (ADHD) and Developmental Coordination Disorder (DCD), are highly heritable, life-long and frequently occurring conditions that may be affected by environmental factors leading to severe impairment of development [1]. NBDs are often characterized by poor social skills, motor and language delays and difficulties in regulating attention and emotion [2]. A recent summary [3] has raised points of convergence and divergence at early developmental stages of ASD and ADHD, as well as similar patterns and overlap of genetic factors in developing clinical symptoms. These findings raised the possibility of a common background or underlying disorder to all NBDs. Although the core deficits of ASD have been considered to involve social functioning [4], retrospective studies indicate that children with ASD exhibit disruption in other developmental domains in the 1st year of life, including motor, attention, and temperament [5]. Retrospective studies are principally based on information from medical record reviews, parent recall, or systematic observational coding of home videotapes taken during the 1st or 2 nd year of life prior to the diagnosis of ASD [6]. To investigate the nature and timing of the emergence of ASD, it has been suggested that prospective, longitudinal studies might be optimal. However, most prospective studies reported thus far focused on infants at high risk, having an older sibling with ASD. These studies indicated that their development in motor, cognitive, language and social domains were grossly intact at 6 months of age, yet, slowing in development was observable when nearing their first birthday $[7,8]$. Noteworthy, a single prospective study on infants at risk for ASD [9] documented abnormal postural control already at 6 months of age.

${ }^{\star}$ Correspondence to: Mina Gurevitz, Maccabi Health Services, Hasharon region, Israel, E-mail: Mina.Gurevich@gmail.com

Key words: neurobehavioral disorders, autism spectrum disorder, attention deficit hyperactivity disorder, developmental coordination disorder, early markers

Received: February 02, 2019; Accepted: February 15, 2019; Published: February 18,2019 
The critical role of motor development and its association with NBD development has been documented. A recent cohort study of healthy term infants linked difficulties in motor development at 3 months of age with cognitive and behavioral difficulties at early school age [10]. Further, the cortical subplate was hypothesized to play a pivotal role in the major neurodevelopmental transformation at $\sim 3$ months of age [11]. In addition, weak motor skills, which traditionally characterize DCD, $[12,13]$ have been frequently found in children with other NBDs. A recent review on the neural basis of DCD has suggested that 'dysfunctional sensor- motor circuits' in the white matter of the brain may lead to motor impairment [14]. These circuits may also include the cortico-spinal tract with lesions in the posterior limb of the internal capsule, which is an important predictor of NBD in children $[15,16]$. Thus, it seems that further prospective studies of infants during their first year of life might shed more light on early signs of NBD development.

The prevalence of ASD and ADHD and awareness to the development of DCD increased over the past two decades [17]. Despite the need to identify a developing disorder at infancy, when brain plasticity enables manipulations, most NBDs are being diagnosed in older children or in teenagers [18]. Evidently, early detection of signs that may predict later development of NBDs is a challenge due to its clinical and practical implications [19], as adequate intervention may improve prognosis [20]. While parents of children with ASD have often noticed developmental problems prior to their first birthday, no screening tool that identifies infants at risk has been established [21]. Such diagnosis is further complicated because a seemingly abnormal sign may rather be associated with extreme behavior [22]. Therefore, the objective of this study was to assess whether thorough analysis of multiple parameters collected from well-baby-care charts of infants may (i) expose early factors that underlie the development of NBDs; (ii) discern factors that predict forthcoming ASD from those predicting forthcoming ADHD or DCD.

\section{Methods}

\section{General information}

Babies in Israel are routinely followed since birth in well-babycare clinics ('Tipat Chalav'), under the supervision of the Ministry of Health, and thus their early medical history is documented. As a pediatrician at the 'Tipat Chalav' clinic of Maccabi Health Services in the Sharon District, the author (MG) meticulously examined, documented, and followed a large number of babies between the years 2000-2010. Access to this dataset enabled MG to search for risk factors at infancy that may predict forthcoming NBDs.

\section{Participants}

For the present study, a sample of 219 files thoroughly documented by MG was acquired from Maccabi dataset. 161 of them were diagnosed for NBD at ages 2-10 years during 2000-2010, in accordance with the DSM-IV criteria [1]: 47 diagnosed for ASD, 56 diagnosed for DCD, and 58 diagnosed for ADHD [23]. The rest 58 children with typical development served as control. The selection of the control children ensured matching of gender and age to the NBD groups.

Premature infants and newborns with major chronic health problems at birth were not included in this study to avoid complications in data analysis.

\section{Covariates}

The following pertinent data were collected: 1. Family history: Parental age and occupation, NBD in siblings, social problems such as divorce or socioeconomic difficulties and parental illness (mental or physical); 2. Perinatal and postnatal history: Gender, Gestational Age (GA), type of delivery, Birth Weight (BW) and Apgar scores; 3. Biometric parameters: Weight, Height and Head Circumference percentiles (Wp, Hp and HCp, respectively); 4. Infant/toddler nutrition: Breast or bottle feeding (extracted breast milk, or formula) in accordance with "The Israeli Ministry of Health guidelines for infant nutrition" (IMH) [24]; 5. Developmental milestone achievement rates: Gross Motor (GM), speech and language and communication evaluations following the Denver Developmental Screening Test (DDST) [25]; 6. Commonly encountered difficulties during the first 3 months: Infant colic and gastroesophageal reflux (GER) [26,27] inferred by the pediatrician and based upon parental description of outbursts of strong cries and recurrent, multiple episodes of spitting-up and/or vomiting; 7. Sleep and feeding problems: Sleep problems were recorded as a difficulty in falling asleep independently, recurrent awakening during the night accompanied by difficulties in resuming sleep, and very short naps during the day [28]. Feeding problems were defined as difficulties in switching from breast feeding to bottle feeding and from breast milk to formula. This also included difficulties upon transition to spoon feeding of solid food, maintaining a daily routine by regulating food amounts, and maintaining constant intervals between feedings; 8 . Child temperament and behavior characteristics: Two extreme types of temperaments, as described by the parents and observed by the pediatrician during the visit, were recognized: The 'difficult child' exhibited anxiety, hysteric crying, restlessness and difficulties upon transitions and changes (Type A). The 'slow to warm-up child' exhibited shyness, embarrassment, introvertedness, isolation and disconnection (Type B) [29,30]; 9. Abnormal findings upon physical examination: The general tone, head and neck control, hyperlaxity of ligaments or any physical or neurological abnormality; 10. Physical co- morbidities: Documented medical illnesses and other diagnoses.

\section{Statistical analysis}

The Unpaired $t$-test was used to compare biometric parameters and social and developmental outcomes of the children in the study and control groups. The Wilcoxon nonparametric test analysis and Pearson Chi-Square analysis were used to compare differential frequencies. A stepwise logistic regression model was used to examine the predictive added power of the independent parameters at each age to predict forthcoming ASD and DCD. Adjusted odds ratios with $95 \%$ confidence intervals were calculated.

\section{Results}

The present study is based on well-baby-care data derived from the general community and thus it differs from numerous previous studies on early markers of ASD, which focused on high- risk infants being siblings of children with ASD.

\section{Family background of participants}

Participant demographic characteristics are shown in Table 1. In the DCD group male predominance was at the highest ratio: 5.2:1 (male/female) vs 3.3:1 in the ASD group and 2.2:1 in the ADHD and Control groups [23].

The data in Table 1 suggest that (i) older parents, especially mothers, are at higher risk to give birth to a child that would develop NBD; (ii) 
Table 1: Demographic characteristics of study groups

\begin{tabular}{|c|c|c|c|c|}
\hline Parameter & $\begin{array}{l}\text { Control } \\
(n=58)\end{array}$ & $\begin{array}{l}\text { ADHD }^{\mathrm{a}} \\
(n=58)\end{array}$ & $\begin{array}{c}\mathbf{A S D}^{\mathrm{b}} \\
(n=47)\end{array}$ & $\begin{array}{c}\mathbf{D C D}^{\mathbf{c}} \\
(n=56)\end{array}$ \\
\hline Male & $65 \%$ & $69 \%$ & $77 \%$ & $\begin{array}{c}84 \% \\
(p=.001)\end{array}$ \\
\hline $\mathrm{GA}^{\mathrm{d}}$ (weeks) & $39.6 \pm 1.1$ & $39.4 \pm 1.4$ & $\begin{array}{c}39.0 \pm 2.1 \\
(\mathrm{p}=.049)\end{array}$ & $\begin{array}{c}38.5 \pm 2.2 \\
(\mathrm{p}=.001)\end{array}$ \\
\hline Delivery (Vaginal/CS ${ }^{\mathrm{e}}$ ) & $77 \% / 23 \%$ & $66 \% / 31 \%$ & $74 \% / 26 \%$ & $68 \% / 32 \%$ \\
\hline $\mathrm{BW}^{\mathrm{f}}(\mathrm{Kg})$ & $3.32 \pm 0.98$ & $3.36 \pm 0.42$ & $\begin{array}{c}3.05 \pm 0.61 \\
(p=.014)\end{array}$ & $\begin{array}{c}3.13 \pm 0.56 \\
(\mathrm{P}=.039)\end{array}$ \\
\hline $\mathrm{HCp}^{\mathrm{g}}(\mathrm{cm})(1-18$ months $)$ & $58.5 \pm 2.51$ & $\begin{array}{c}46.1 \pm 3.76 \\
(p=.007)\end{array}$ & $\begin{array}{c}47.6 \pm 3.74 \\
(p=.019)\end{array}$ & $\begin{array}{c}44.5 \pm 3.65 \\
(\mathrm{p}=.002)\end{array}$ \\
\hline Apgar (1 min) & $8.98 \pm 0.30$ & $8.75 \pm 0.95$ & $8.73 \pm 0.20$ & $\begin{array}{c}8.40 \pm 0.40 \\
(p=.003)\end{array}$ \\
\hline Apgar (5 min) & $9.95 \pm 0.23$ & $9.93 \pm 0.26$ & $9.86 \pm 0.09$ & $\begin{array}{c}9.30 \pm 0.15 \\
(\mathrm{p}=.002)\end{array}$ \\
\hline Maternal age (y) & $30.74 \pm 3.8$ & $\begin{array}{c}32.9 \pm 6.04 \\
(p=.024)\end{array}$ & $31.7 \pm 3.01$ & $\begin{array}{c}32.7 \pm 4.8 \\
(p=.023)\end{array}$ \\
\hline Paternal age (y) & $34.20 \pm 5.06$ & $\begin{array}{c}36.7 \pm 6.73 \\
(p=.027)\end{array}$ & $34.7 \pm 3.80$ & $35.8 \pm 5.40$ \\
\hline Maternal academic occupation & $62 \%$ & $\begin{array}{c}39 \% \\
(p=.022)\end{array}$ & $63 \%$ & $52 \%$ \\
\hline Paternal academic occupation & $60 \%$ & $\begin{array}{c}33 \% \\
(p=.007)\end{array}$ & $73 \%$ & $66 \%$ \\
\hline $\mathrm{NBDs}^{\mathrm{h}}$ in family & 0 & $\begin{array}{c}15 \% \\
(p=.001)\end{array}$ & $10 \%$ & $7 \%$ \\
\hline Social difficulties in family & $1.7 \%$ & $\begin{array}{c}15.5 \% \\
(p=.008)\end{array}$ & 0 & $5 \%$ \\
\hline $\begin{array}{l}\text { Breast / Formula } \\
>6 \text { months nursing }\end{array}$ & $\begin{array}{c}64 \% / 36 \% \\
19 \%\end{array}$ & $\begin{array}{c}40 \% / 60 \% \\
12 \%\end{array}$ & $\begin{array}{c}55 \% / 45 \% \\
19 \%\end{array}$ & $\begin{array}{c}71 \% / 29 \% \\
32 \%\end{array}$ \\
\hline $\begin{array}{c}\text { Neonatal } \\
\text { Complications }\end{array}$ & $12 \%$ & $5 \%$ & $13 \%$ & $23 \%$ \\
\hline
\end{tabular}

The numbers presented are averages \pm standard errors. Upon significant difference from control the $p$ value is provided. The data in the ADHD column is derived from ref. 23. (a: Attention Deficit Hyperactivity Disorder; b: Autistic Spectrum Disorder; c: Developmental Coordination Disorder; d: Gestational Age; e: Cesarean Section; f: Birth Weight; g: Head Circumference percentile; h: Neurobehavioral Disorder)

children with ASD are found at higher frequency in families with paternal higher education; (iii) NBDs, develop more frequently in families with socioeconomic difficulties and familial history of these disorders.

\section{Perinatal history}

The average GA, BW and HCp of children with ASD or DCD were significantly lower from those of children in the control group (Table 1). Apgar scores were significantly lower only in the DCD group in comparison to the other groups. Most children with DCD were breast fed (71\%) and 32\% of them were nursed for over 6 months, Noteworthy, the weight and HC of prolonged breast-fed children followed their trajectories, which likely limited excess weight gain.

\section{Biometric parameters}

Comparison of the biometric parameters HCp (head circumference percentile), Wp (weight percentile) and Hp (height percentile) of the ASD and DCD groups at 1, 3, 9 and 18 months of age with those of the control group revealed significant differences. The parameters of typically developing infants (control group) have proportionally changed along growth, and so were the parameters of the DCD infants, although at a lower scale. In contrast, the changes in these parameters in the ASD group have shown substantial disproportional variations (Table 2). The Hp values in the ASD group were similar to those of the control group, but their Wp values at 3, 9 and 18 months were significantly lower. The HCp values were lower than their control equivalents at 1 and 3 months, but similar to those of the control group at 9 and 18 months of age. Yet, careful inspection of the data indicated that the differences in HC growth rates among the groups depended on the weight and head circumference values at birth (BW and $\mathrm{HC}$ ). Therefore, the study groups were divided into three subgroups according to the BW percentile (below 10p $=$ subgroup A; between 11-50p = subgroup B; and above 51p = subgroup C), and reanalysed.

Figure 1, divided according to the subgroups A, B and C, demonstrates the varying Wp and HCp values since birth to 18 months of age of the ASD group in comparison to an ADHD group (derived from ref. 23). Clearly, the HCp values of the ASD infants increased when the BW was either below 10p or between 11-50p (Figures 1A and B). However, when the BW was high, the HCp values at 0-18 months of age have slightly decreased (Figure 1C). Thus, the HCp values at infancy of those born with high BW cannot be considered an early sign for the development of ASD. In contrast to the ASD infants, the HCp values of ADHD infants decreased in subgroups $B$ and $C$, but not in subgroup $\mathrm{A}$, and thus the HCp values at infancy of those born with BW below $10 \mathrm{p}$ cannot serve as an early sign of ADHD development. Noteworthy, the HCp values of the DCD infants generally followed their trajectories although at lower values compared to the control (not shown).

\section{Developmental milestones and physical findings}

A significant delay in motor development was observed in NBD infants at 9 and 18 months of age (Table 3).

The children with DCD have shown delays even sooner, at 3 months of age. Abnormal physical findings were observed at high frequencies in infants who were later diagnosed for ASD or DCD 
Table 2: Biometric parameters of the study groups in comparison to the control. Number of participants appears in parenthesis. Each value is the average percentile \pm standard error. $p$ designates significance of deviation from control

\begin{tabular}{|c|c|c|c|c|c|}
\hline Patient & Control & ASD & $p$ & DCD & $p$ \\
\hline $\mathrm{HCp}^{\mathrm{a}} 1 \mathrm{mb}$ & (56) $57.5 \pm 4.3$ & (37) $44.9 \pm 4.4$ & .024 & (53) $46.3 \pm 3.8$ & .029 \\
\hline $\mathrm{HCp} 3 \mathrm{~m}$ & (58) $64.5 \pm 2.5$ & (39) $46.2 \pm 4.1$ & $<.001$ & (55) $45.5 \pm 3.8$ & $<.001$ \\
\hline HCp 9m & (58) $56.5 \pm 2.7$ & (39) $48.7 \pm 4.2$ & $\mathrm{NS}^{\mathrm{e}}$ & (55) $43.0 \pm 3.9$ & .005 \\
\hline HCp $18 \mathrm{~m}$ & (58) $54.9 \pm 2.8$ & (35) $53.3 \pm 4.2$ & NS & (55) $44.5 \pm 4.3$ & .042 \\
\hline $\mathrm{Wp}^{\mathrm{c}} 1 \mathrm{~m}$ & (56) $49.0 \pm 3.4$ & (37) $45.7 \pm 4.7$ & NS & (56) $40.6 \pm 3.6$ & NS \\
\hline Wp 3m & (58) $70.1 \pm 2.2$ & (39) $52.9 \pm 4.5$ & $<.001$ & (55) $47.9 \pm 3.6$ & $<.001$ \\
\hline Wp 9m & (58) $58.5 \pm 2.9$ & (39) $46.9 \pm 4.8$ & .031 & (55) $38.2 \pm 3.8$ & $<.001$ \\
\hline $\mathrm{Wp} 18 \mathrm{~m}$ & (58) $61.2 \pm 2.9$ & (35) $45.5 \pm 5.2$ & .006 & (55) $37.6 \pm 3.6$ & $<.001$ \\
\hline $\mathrm{Hp}^{\mathrm{d}} 1 \mathrm{~m}$ & (11) $46.8 \pm 7.2$ & (34) $43.2 \pm 5.0$ & NS & (37) $42.9 \pm 4.6$ & NS \\
\hline Hp 3m & (58) $61.2 \pm 2.6$ & (39) $50.0 \pm 4.6$ & .026 & (55) $47.1 \pm 3.6$ & .002 \\
\hline Hp 9m & (58) $58.7 \pm 3.2$ & (39) $54.3 \pm 4.4$ & NS & (55) $47.8 \pm 3.8$ & .030 \\
\hline Hp 18m & (58) $57.9 \pm 3.5$ & (35) $54.1 \pm 5.0$ & NS & (55) $44.8 \pm 3.5$ & .012 \\
\hline
\end{tabular}

(a: Head Circumference percentile; b: Months; c: Weight percentile; d: Height percentile; e: Not significant)

Table 3: Frequencies of various difficulties during infancy among children of the ASD, DCD and Control groups. $p$ designates significance

\begin{tabular}{|c|c|c|c|c|c|c|}
\hline $\begin{array}{c}\text { Parameter } \\
\text { (difficulty or delay) }\end{array}$ & $\begin{array}{c}\text { Age } \\
\text { (months) }\end{array}$ & $\begin{array}{c}\text { Control } \\
(n=58)\end{array}$ & $\begin{array}{c}\text { ASD } \\
(n=42)\end{array}$ & $p$ & $\begin{array}{l}\text { DCD } \\
(n=56)\end{array}$ & $p$ \\
\hline Sleep & 3 & 0 & $14 \%$ & .004 & $16 \%$ & .001 \\
\hline Feeding & $\begin{array}{c}3 \\
9 \\
18\end{array}$ & $\begin{array}{c}2 \% \\
10 \% \\
7 \%\end{array}$ & $\begin{array}{l}17 \% \\
17 \% \\
12 \%\end{array}$ & $\begin{array}{l}.009 \\
N^{b} \\
\text { NS }\end{array}$ & $\begin{array}{l}23 \% \\
25 \% \\
20 \%\end{array}$ & $\begin{array}{l}<.001 \\
.034 \\
.040\end{array}$ \\
\hline Motor development & $\begin{array}{c}3 \\
9 \\
18 \\
\end{array}$ & $\begin{array}{c}19 \% \\
14 \% \\
2 \%\end{array}$ & $\begin{array}{l}31 \% \\
38 \% \\
21 \%\end{array}$ & $\begin{array}{l}\mathrm{NS} \\
.005 \\
.002\end{array}$ & $\begin{array}{l}50 \% \\
39 \% \\
21 \%\end{array}$ & $\begin{array}{l}<.001 \\
.002 \\
.001\end{array}$ \\
\hline Communication & $\begin{array}{c}3 \\
9 \\
18 \\
\end{array}$ & $\begin{array}{l}0 \\
0 \\
0\end{array}$ & $\begin{array}{l}12 \% \\
9.5 \% \\
31 \%\end{array}$ & $\begin{array}{c}.011 \\
.029 \\
<.001\end{array}$ & $\begin{array}{l}0 \\
0 \\
0\end{array}$ & $\begin{array}{l}\text { NS } \\
\text { NS } \\
\text { NS }\end{array}$ \\
\hline Speech \& language & $\begin{array}{c}9 \\
18\end{array}$ & $\begin{array}{l}2 \% \\
9 \%\end{array}$ & $\begin{array}{l}24 \% \\
64 \%\end{array}$ & $\begin{array}{l}.001 \\
.001\end{array}$ & $\begin{array}{l}9 \% \\
30 \%\end{array}$ & $\begin{array}{l}\text { NS } \\
.004\end{array}$ \\
\hline $\begin{array}{l}\text { Infant colic } \\
\text { GER }^{\mathrm{a}}\end{array}$ & $\begin{array}{l}3 \\
9\end{array}$ & $\begin{array}{c}9 \% \\
10 \%\end{array}$ & $\begin{array}{l}11 \% \\
16 \%\end{array}$ & $\begin{array}{l}\text { NS } \\
\text { NS }\end{array}$ & $\begin{array}{l}18 \% \\
16 \%\end{array}$ & $\begin{array}{l}\text { NS } \\
\text { NS }\end{array}$ \\
\hline
\end{tabular}

(a: Gastroesophageal Reflux; b: Not significant)

Table 4. Comparison of the frequencies of physical abnormalities during infancy among the ASD, DCD and Control children. $p$ designates significance

\begin{tabular}{|c|c|c|c|c|}
\hline Physical abnormalities & $\begin{array}{c}\text { Control } \\
(n=58)\end{array}$ & $\begin{array}{c}\text { ASD } \\
(n=39)\end{array}$ & $\boldsymbol{p}$ & $\begin{array}{c}\text { DCD } \\
(n=55)\end{array}$ \\
\hline Laxity of ligaments & $19.0 \%$ & $33.3 \%$ & NS $^{\mathrm{b}}$ & $52.7 \%$ \\
\hline $\mathrm{DP}^{\mathrm{a}}$ & $3.4 \%$ & $28.2 \%$ & $<.001$ & $30.9 \%$ \\
\hline Torticollis & $3.4 \%$ & $7.7 \%$ & $\mathrm{NS}$ & $18.2 \%$ \\
\hline Hypertonia & $6.9 \%$ & $30.8 \%$ & .002 & $25 \%$ \\
\hline Hypotonia & $1.7 \%$ & $30.8 \%$ & $<.001$ \\
\hline
\end{tabular}

(a: Deformational Plagiocephaly; b: Not significant)

(Table 4). Thus, the development of NBDs seems to be associated with physical abnormalities and difficulties in regulation of muscle tone and ligaments since birth. This is later manifested as a delay in the development of motor milestones.

\section{Communication difficulties}

Communication difficulty, one of the core symptoms of ASD, was observed at 3, 9 and 18 months in $12 \%(p=.011), 9.5 \%(p=.029)$ and $31 \%(p=.000)$, respectively, of the infants later diagnosed for ASD (Table 3).

\section{Sleeping and feeding problems}

The NBD children experienced during infancy more difficulties in self-regulation than children of the control group (Table 3). At 3 months of age, $12-16 \%$ of all NBD children had sleep problems. At 3, 9 and 18 months of age, feeding difficulties were reported in $23 \%(p<.001)$, $25 \%(p=.034)$ and $20 \%(p=.040)$, respectively, of the DCD children, whereas feeding problems were reported in $17 \%$ of the ASD children only at 3 months of age $(p=.009)$.

\section{Behavioral characteristics}

Behavioral characteristics during infancy as described by the parents (39 of the ASD and 55 Children of the DCD groups) and documented by the pediatrician during the well-baby-care visits were compared among the study groups. Type A babies were characterized by Anxiety, Hysteric cry, Restlessness, Adaptation and Transition difficulties, whereas Type B babies were characterized by Shyness, Embarrassment, Introvertedness, Isolation and Disconnection. $74 \%$ of the ASD children were described at infancy as Type A and $26 \%$ as Type B in comparison to $52 \%$ Type A and only $9 \%$ Type B of the DCD children.

\section{Physical co-morbidities}

As is shown in Table 5, children diagnosed for ASD and DCD had a history of recurrent or chronic illnesses, such as allergy related problems, 
A

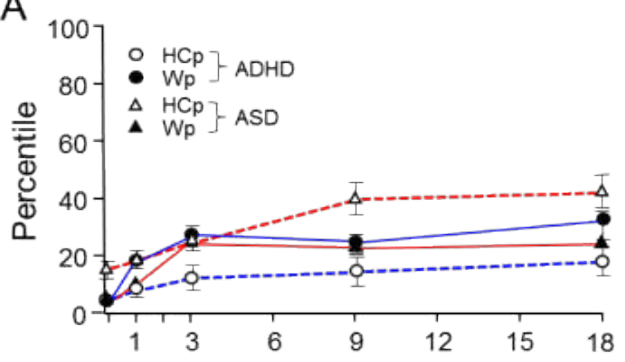

B

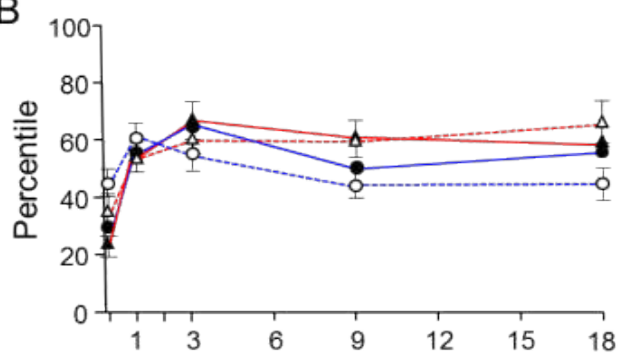

C

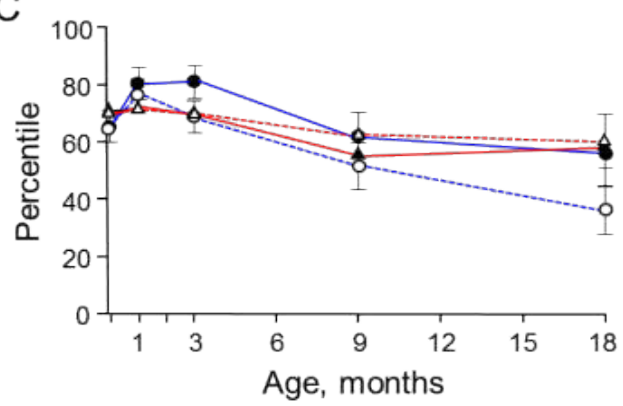

Figure 1. Alterations in HCp and Wp during the first 18 months of life in ASD and ADHD high risk infants (Data of ADHD high risk infants is taken from ref. 23). A, Birth weight (BW) below $10^{\text {th }}$ percentile; B, BW between $11^{\text {th }}$ to $50^{\text {th }}$ percentiles; C, BW above $51^{\text {th }}$ percentile. HCp curves are designated by dashed lines and Wp by continuous lines. ASD in red and ADHD in blue. Each point provides the average value \pm standard error. For clarity, the data of control and DCD high risk infants is not presented as both groups have not shown significant alterations in their Wp and $\mathrm{HCp}$ trajectories

Table 5. Frequencies of physical co-morbidities among children of the ASD and DCD groups

\begin{tabular}{|l|c|c|}
\hline Parameter & $\begin{array}{c}\text { ASD } \\
(n=39)\end{array}$ & $\begin{array}{c}\text { DCD } \\
(n=55)\end{array}$ \\
\hline Milk allergy, Eosinophilia, Asthma, Atopic Dermatitis & $41 \%$ & $38 \%$ \\
\hline $\begin{array}{l}\text { Recurrent Otitis Media, Serous Otitis Media, hearing loss, } \\
\text { Adenoidectomy, Ventilation tubes insertion }\end{array}$ & $31 \%$ & $27 \%$ \\
\hline Vision problems, Strabismus, Eye glasses & $23 \%$ & $7 \%$ \\
\hline Recurrent trauma and accidents & $15 \%$ & $7 \%$ \\
\hline Recurrent illness & $13 \%$ & $7 \%$ \\
\hline Constipation & 0 & $11 \%$ \\
\hline
\end{tabular}

hearing problems (e.g., ear infections and Adenoid hypertrophy) and vision problems (e.g., far- or short-sided and strabismus), which required surgical interventions. Comparison of the other parameters revealed twice as frequent recurrent trauma and illness in children with ASD. The data of this table suggests that children with NBD would often exhibit disturbances at the immune system.

\section{Statistical analysis}

Stepwise regression analyses were conducted to trace among all parameters those that may predict a forthcoming ASD or DCD. Final analysis was performed with the background parameters used in the first step, followed by the best clinical predictors and using younger ages in earlier steps and older ages in the final steps. Adjusted odds ratios with $95 \%$ confidence intervals were calculated. Six parameters have been found to predict an increased risk for development of ASD: At birth- small HCp (OR: 0.98; 95\% CI: 0.97, 1.000; p 0.034); At 3 months- feeding problems (OR: 16.8; 95\% CI: 1.9, 149; p 0.012) and low Wp (OR: 0.93; 95\% CI: 0.943, 0.984; p 0.000); At 9 months- motor development difficulties (OR: 5.65; 95\% CI: 1.76,18.15; p 0.004) and speech developmental delay (OR: 11.76; 95\% CI: 1.16, 120; p 0.037); and at 18 months- motor development difficulties (OR: 41.03; 95\% CI: 3.38, 499; p 0.004) and communication developmental delay (OR: 78.5; 95\% CI: 6.84, 902; p 0.000). Four parameters have been found to predict the development of DCD: Early gestational age (OR: $0.64 ; 95 \%$ CI: 0.482 , 0.861; p 0.003); feeding problems (OR: 12.96; 95\% CI: 1.26 , 132.8; p 0.031) and low Wp (OR: 0.957; 95\% CI: 0.937, 0.977; p 0.000) with motor development difficulties (OR: 5.364; 95\% CI: 1.982, 14.5; p $0.001)$ at 3 months. Motor development difficulties were also predictive of DCD at 9 months (OR: 5.35; 95\% CI: 1.847, 15.5; p 0.002) and at 18 months (OR: 13.03; 95\% CI: 1.497, 113.34; p 0.02).

Multivariate regression model: Serial regression analyses were conducted to trace the best predictor for ASD and DCD at each age, while analyses regarding the ADHD group were previously reported [23]. Final analysis was performed as described above. At birth, GA and BW account for $69 \%$ of the variance in the ASD group and $42 \%$ of the variance in the DCD group. HC at 1 month of age accounts for $5 \%$ of the variance in the ASD group and $4 \%$ in the DCD group. At 3 months of age, biometric parameters ( $\mathrm{HCp}, \mathrm{Wp}$ and $\mathrm{Hp}$ ), sleep and feeding problems and communication difficulties account for $35 \%$ of the variance in the ASD group. The same parameters in addition to motor developmental delay account for $36 \%$ of the variance in the DCD group. At 9 months of age, Wp, motor developmental delay and speech and language delays account for $24 \%$ of the variance in the ASD group. At 18 months of age, Wp, motor developmental delay, speech and language delays and communication problems account for $38 \%$ of the variance in the ASD group. The same parameters (except for motor developmental delay) account for $29 \%$ of the variance in the DCD group. At 9 and 18 months of age, biometric parameters (HCp, Wp and $\mathrm{Hp}$ ), feeding problems and motor developmental delay account for $26 \%$ and $27 \%$, respectively, of the variance in the DCD group. The overall predictive model for ASD accounts for $85 \%$ of the variance explained and the overall predictive model of DCD accounts for $71 \%$ of the variance explained.

\section{Discussion}

The results of this study suggest that infants with high probability to develop neurobehavioral disorders (NBDs), here named 'high risk infants', exhibit worrisome clinical and physical signs during the first year of life, which may be used as early predictors. Interestingly, ASD and DCD, as well as ADHD [23] exert similar risk factors during the first months in life, such as early gestational age (GA), low weight percentile $(\mathrm{Wp})$, feeding difficulties (as reflected on deviations from weight and head circumference percentile trajectories), and difficulties in motor development. Still, a few early parameters appear to specify the development of certain NBDs. Speech delay specifies the development of ASD and ADHD [23], while delay in communication skills seems to specify ASD development.

The present study corroborates the proposed link between rapid weight gain and NBD development and also emphasizes the critical 
timing and extent of these changes, which determine the type of NBD that may develop. Two parameters that mostly affect the weight and $\mathrm{HC}$ growth rates during infancy are GA and BW. The division of the study groups to 3 subgroups according to the $\mathrm{BW}(<10 \mathrm{p}, 11-50 \mathrm{p}$ and $>51 \mathrm{p})$ was instrumental in showing the differences in the correlation between $\mathrm{Wp}$ and HCp changes in infants with high probability to develop NBD. Rapid increase in weight (intrauterine and/or during the first 3 months) followed by a decrease in HCp may discern the development of ADHD from that of ASD. It is shown that in infants with BW below average (Figure $1 \mathrm{~A}$ and $\mathrm{B}$ ) the rapid weight gain was accompanied by an increase in $\mathrm{HC}$ growth rate.

Moreover, the higher the increase in $\mathrm{Wp}$ at 3 months, more severe was the NBD that developed ( 2.8 fold for ASD, $\sim 2.4$ fold for ADHD and $\sim 2.2$ fold for DCD). It is plausible that the high BW and $\mathrm{HC}$ at birth in subgroup $\mathrm{C}$ occurred during the last trimester of pregnancy. Hence, the claim that an increase of $\mathrm{HC}$ characterizes infants that may develop ASD [31] better be refined according to the BW and weight gain rates.

The above results have drawn attention to the importance of weight gain rates at infancy and the development of NBDs. This link is also reflected on two major problems, obesity [32] and NBDs [17]. An association between parental obesity and NBD development in offspring has been recently suggested. Maternal metabolic conditions during pregnancy and obese fathers were described as risk factors for ASD and other NBDs [33,34]. The link between obesity and ASD raised the possibility of a common genetic background, such as deletions in chromosome 16p11.2 [35,36]. Another suggestion linked epigenetic obesity with induced changes in DNA modifications (e.g., methylation), thus affecting gene expression and regulation [37]. A study of a nationally representative sample of school-aged children linked lesions in the non-dominant right hemisphere and the parietal lobe of children with excessive weight gain to abnormal brain functioning (cognitive impairment in visuo-spatial organization and general mental ability) [38]. The putative connection between excessive weight and brain damage could involve increased body status, such as low-degree inflammation of blood vessels in the brain since overweight has been identified as a pro-inflammatory state [39]. In addition, hyper-insulinemia has been shown to be associated with hampered glucose metabolism and insulin signaling at several brain regions (e.g., frontal lobes, hippocampus) involved in planning and organizing [40]. Therefore, rapid increase in weight within 1-3 month of age may incite metabolic and hormonal changes that affect the brain and lead to the development of NBDs.

In contrast to the ASD and ADHD [23] 'high risk infants', the DCD 'high risk infants' have shown the utmost deviation in GA and BW, yet they followed their low Wp and HCp trajectories till 18 months of age (Table 2). Therefore, the search for early DCD risk factors has focused on motor difficulties, one among the several early markers of NBD development. Indeed, this study reveals a link between irregular motor development as well as deviations from normal milestone achievement rates and the development of NBDs. The earliest prevalent physical signs in 'high risk infants' are hypertonia or hypotonia, stiffness or hyperlaxity of joints, and Torticollis or Deformational Plagiocephaly (DP), all affecting motor development (Table 4). Indeed, a dramatic increase in referrals for assessment and treatment of deformational plagiocephaly (DP) can be observed during the last two decades pertinent to the "Back To Sleep" guidelines as a preventive measure of SIDS (Sudden Infant Death Syndrome) [41]. Although considered a medically benign condition, DP may be associated with developmental difficulties, suggesting that children with this condition should be monitored for developmental delays or deficits [42]. Another prospective study on infants at risk has shown significantly poorer fine and gross motor scores at 36 months of age in those later diagnosed for ASD [43] thus concluding that early motor difficulties may predict a forthcoming NBD.

A wide range of motor delays and deficits that constitute the core of DCD development have also been reported in ASD and ADHD patients, and there is a link between early deviations from motor developmental trajectories and psychological outcomes at childhood [44]. The numerous motor developmental irregularities (Table 3) and physical abnormalities (e.g., DP, Torticollis, Hyperlaxity; Table 4) that typify infants at risk to develop NBDs may assist in early identification of a specific NBD and the establishment of a specific intervention program based on physical training for improving motor, cognitive and behavioral development. DCD infants born with significantly lower Apgar scores at 1 and 5 minutes also reveal higher incidence of neonatal complications in comparison to the other study groups (Table 1). These early signs may be attributed to lower GA or higher incidence of lax ligaments and asymmetry in position (Table 4), which might be considered minor among the predictive parameters for forthcoming DCD. Another significant observation is the relationship between infant nutrition and growth rate. The weight and $\mathrm{HC}$ of prolonged breast-fed infants, who later developed DCD, followed their trajectories (Tables 1 and 2). Hence, prolonged breast milk feeding may limit excessive weight gain.

Minimal eye contact (communication delay), which serves as a main ASD specific clinical marker [1], was observed in the present study at an early stage ( 3 months) only in $12 \%$ of the ASD group and at 18 months in $31 \%$ of the group (Table 3 ). Thus, although this marker has been considered significant in ASD diagnosis, it seems from the present study that it is actually not a sufficiently sensitive marker at early infancy. Other common symptoms detected at elevated frequencies in 'high risk infants' are difficulties in controlling sleep, feeding (Table 3) and behavior. Although these predictive markers are detectable during the first year of life, they are often being overlooked by both parents and primary care pediatricians due to the difficulty to discern problems of extreme behavior from early signs of a developing NBD. This study highlights a number of early markers for NBD development and supports the hypothesis about an early common pathway that later diverges to ASD, ADHD and DCD. The findings of this work point to abnormal motor developmental factors as the main early common signs for all NBDs, which are combined with difficulties in regulating processing of sensory stimulations affecting feeding, sleeping and behavior. The end result of such combination is rapid weight gain accompanied by deviations from head growth trajectory during the first three months of life. The extent of deviation may discriminate among NBDs. The most severe increase in $\mathrm{HC}$ would suggest a forthcoming ASD, whereas the least severe alteration from trajectory would suggest a forthcoming DCD.

Additional points raised in the present study pertain to (i) the high number of NBD children diagnosed for allergic disorders ( $40 \%$, Table 5), as was also reported in a large scale study on the prevalence of allergic co-morbidities (e.g., asthma, allergic rhinitis, atopic dermatitis, and allergic conjunctivitis) in NBD children [45]; (ii) the high number of NBD children also suffering from hearing problems, due to fluid accumulation in the ears, and sleeping problems, due to enlargement of adenoids causing obstructive sleep apnea. Notably, $\sim 30 \%$ of the ASD and DCD children went through adenoidectomy and Ventilation Tubes insertion. The link between attention deficit, hyperactivity 


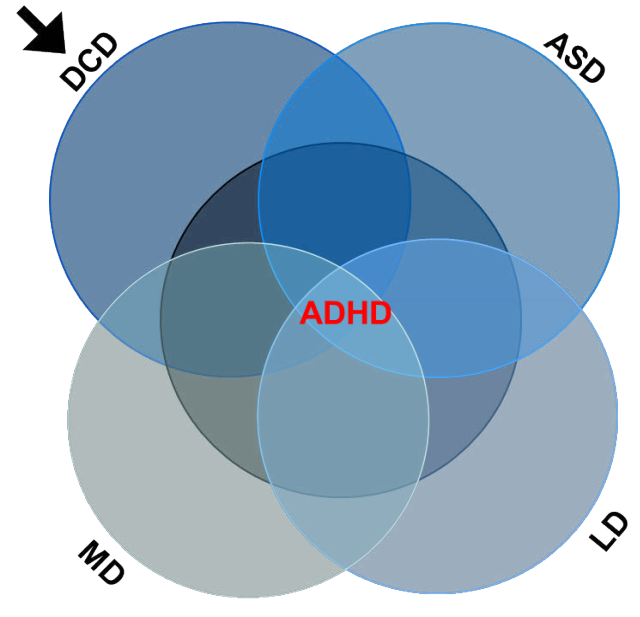

Figure 2. Schematic description of NBDs overlapping and its hypothetical implication. ADHD is centered due to its highest incidence. Besides similar characteristics at early stages of development, the various disorders also reveal common predictive markers (e.g., motor development delay; rapid weight gain affecting head circumference; and difficulties in sensory regulation). Thus, intervention during 0-3 months of age in infants with predictive factors for developing a certain NBD might diminish the development of all other NBDs. This hypothesis is mostly valid for prevention of DCD (designated by the arrow) mainly by motor intervention

and impulsivity symptoms and airway obstruction due to AdenoTonsillar hypertrophy has been previously reported [46]. Therefore, infants exhibiting parameters for a developing NBD should be evaluated for physical co-morbidities (e.g., allergy, hearing difficulty, adenoid hypertrophy) as early as possible and be given the proper treatment. Long term follow-up of the children of the study groups revealed high percentage learning disabilities (LD) and mental disorders (MD) at school age. These late effects in addition to early markers, such as rapid weight gain and difficulties in regulation of fundamentals like motor development, eating and sleeping, which typify ASD, ADHD and DCD infants, raise the possibility of a common early background in the development of all NBDs. The link among ASD, ADHD, DCD, LD and MD has been previously proposed [47]. The common background among the various NBDs is depicted schematically in Figure 2, with ADHD centered due to its highest incidence. This scheme suggests that early treatment of one disorder, especially DCD, would affect the development of all other NBDs (see arrow in Figure 2). The hypothesis here raised is that early prevention of DCD development in 'high risk infants' might establish brain connectivity that would diminish the risk for development of NBDs. It seems, though, that infants with one disorder should be routinely checked for the other disorders.

\section{Conclusions}

1.The findings of this study establish an initial directory of early risk factors that might be used to predict emerging NBDs at infancy. Although further analysis using a larger sample of infants would substantiate the results, this is for the first time that correlation between the increase in HCp and excessive weight gain at early infancy linked to NBD development is being proposed.

2. The correlation between rapid increase in weight and the development of NBDs suggests that prevention of intrauterine and infant rapid gain in weight might, among other precautions, be critical in diminishing the number of children with ASD. Since brain plasticity at infancy enables manipulations to strengthen motor development and the corresponding neural functions, the sooner a risk factor is recognized in a baby, more efficient an early tailored bio-psycho-social treatment might be.

\section{Declarations}

\section{Ethics approval and consent to participate}

The study was performed using patients charts and was approved by the Ethics Committee of Maccabi Health Services, Israel.

\section{Consent for publication}

No consent form was required by this Committee. Not applicable

\section{Availability of data and materials}

The dataset used and analysed during this study is available from the author on reasonable request.

\section{Conflicts of interest}

The author declares that she has no competing interests.

\section{Funding}

Not applicable

\section{Authors' contribution}

The author performed this research and wrote the manuscript.

\section{Acknowledgements}

Special thanks to Maya Varon and Ilana Gelerenter for the statistical analyses.

\section{References}

1. American Psychiatric Association (2000) Diagnostic and Statistical Manual of Mental Disorders, Text Revision (DSMIV-TR) 4th Edition. Am Psychol Assoc Washington DC.

2. Mulligan A, Anney RJ, O'Regan M, Chen W, Butler L et al. (2009) Autism symptoms in attention- deficit/hyperactivity disorder: a familial trait which correlates with conduct, oppositional defiant, language and motor disorders. J Autism Develop Disorders 39: 197-209.

3. Johnson MH, Gliga, T, Jones E, Charman T (2015) Annual Research Review: Infant development, autism, and ADHD - early pathways to emerging disorders. $J$ Child Psychol Psychiat 56: 228-247.

4. Pelphrey KA, Carter EJ (2008) Charting the typical and atypical development of the social brain. Develop Psychopathol 20: 1081-1102.

5. Werner E, Dawson G, Munson J, Osterling J (2005) Variation in early developmental course in autism and its relation with behavioral outcome at 3-4 years of age. J Autism Develop Disorders 35: 337-350.

6. Luyster R, Richler J, Risi S, Hsu WL, Dawson G et al. (2005) Early regression in social communication in autism spectrum disorders: A CPEA study. Develop Neuropsychol 27: 311-336.

7. Landa R, Garrett-Mayer E (2006) Development in infants with autism spectrum disorders: A prospective study. J Child Psychol Psychiat 47: 629-638.

8. Ozonoff S, Iosif AM, Baguio F, Cook IC, Hill MM et al. (2010) A prospective study of the emergence of early behavioral signs of autism. J Am Acad Child Adolesc Psychiat 49: 256-266.

9. Flanagan JE, Landa R, Bhat A, Bauman M (2012) Head lag in infants at risk for autism: a preliminary study. Am J Occup Ther 66: 577-585. [Crossref]

10. Hitzert MM1, Roze E, Van Braeckel KN, Bos AF (2014) Motor development in 3 -month-old healthy term-born infants is associated with cognitive and behavioural outcomes at early school age. Dev Med Child Neurol 56: 869-876. [Crossref]

11. Hadders-Algra M (2007) Putative neural substrate of normal and abnormal general movements. Neurosci Biobehav Rev 31: 1181-1190.

12. Blank R, Smits-Engelsman B, Polatajko H, Wilson P (2012) European Academy for Childhood Disability (EACD): recommendations on the definition, diagnosis and intervention of developmental coordination disorder (long version). Develop Med Child Neurol 54: 54-93. 
13. Zwicker JG, Missiuna C, Harris SR, Boyd LA (2012) Developmental coordination disorder: a review and update. Eur J Paediatr Neurol 16: 573-581. [Crossref]

14. Peters LH, Maathuis CG, Hadders-Algra M (2013) Neural correlates of Developmental Coordination Disorder. Develop Med Child Neurol 55: 59-64.

15. Rutherford MA, Pennock JM, Counsell SJ, Mercuri E, Cowan FM, et al. (1998) Abnormal magnetic resonance signal in the internal capsule predicts poor neurodevelopmental outcome in infants with hypoxic-ischemic encephalopathy. Pediatrics 102: 323-328. [Crossref]

16. Jeste SS (2011) The neurology of autism spectrum disorders. Curr Opin Neurol 24 132-139. [Crossref]

17. Center for Disease Control and Prevention (2012) Autism and Developmental Disability Monitoring Network Surveillance year 2010. Prevalence of Autism Spectrum Disorder Among Children Aged 8 Years - Autism and Developmental Disabilities Monitoring Network, 11 Sites, United States, 2010. MMWR Surveillance Summary 61: 1-19.

18. Dawson G (2008) Early behavioral intervention, brain plasticity and the prevention of autism spectrum disorder. Develop Psychopathol 20: 775-803.

19. Pierce K, Carter C, Weinfeld M, Desmond J, Hazin R, et al. (2011) Detecting, studying, and treating autism early: the one-year well-baby check-up approach. J Pediatr 159 : 458-465. [Crossref]

20. Rogers SJ, Vismara LA (2008) Evidence-based comprehensive treatments for early autism. J Clinic Child Adolesc Psychol 37: 8-38.

21. Steiner AM, Goldsmith TR, Snow AV, Chawarska K (2012) Practitioner's guide to assessment of autism spectrum disorders in infants and toddlers. J Autism Dev Disord 42: 1183-1196. [Crossref]

22. Wetherby AM, Brensan-Maddox S, Peace V, Newton L (2008) Validation of the InfantTodler checklist as a broadband screener for autism spectrum disorders from 9 to 24 months of age. Autism 12: 487-512.

23. Gurevitz M, Geva R, Varon M, Leitner Y (2014) Early markers in infants and toddlers for development of ADHD. J Attention Disorders 18: 14-22.

24. Ministry of Health Israel (2009) Infant nutrition guidelines. Ministry of Health, Public Health Department, Government of Israel (http://www.health.gov.il). 2009.

25. Frankenburg WK, Dodds JB (1967) The Denver developmental screening test. $J$ Pediatr 71: 181-191. [Crossref]

26. Roberts DM, Ostapchuk M, O'Brien JG (2004) Infantile colic. Am Fam Physician 70: 735-740. [Crossref]

27. Rudolph CD, Mazur LJ, Liptak GS, Baker RD, Boyle JT et al. (2001) Guidelines for evaluation and treatment of gastroesophageal reflux in infants and children: Recommendations of the North American Society for Pediatric Gastroenterology and Nutrition. J Pediatric Gastroenterol Nutrition 32: S1-S31.

28. White BP, Gunnar MR, Larson MC, Donzella B, Barr RG (2000) Behavioral and physiological responsivity, sleep, and patterns of daily cortisol production in infants with and without colic. Child Dev 71: 862-877. [Crossref]

29. Slining MM, Adair L, Goldman BD, Borja J, Bentley M (2009) Infant temperament contributes to early infant growth: A prospective cohort of African American infants. Jntern J Behav Nutrit Phys Activ 6: 51-61.

30. McDevitt SC, Carey WB (1978) The measurement of temperament in 3--7 year old children. J Child Psychol Psychiatry 19: 245-253. [Crossref]
31. Courchesne E, Carper R, Akshoomoff N (2003) Evidence of brain overgrowth in the first year of life in autism. JAMA 290: 337-344. [Crossref]

32. Ogden CL, Carroll MD, Kit BK, Flegal KM (2014) Prevalence of childhood and adult obesity in the United States, 2011-2012. JAMA 311: 806-814. [Crossref]

33. Krakowiak P, Walker CK, Bremer AA, Baker AS, Ozonoff S, et al. (2012) Maternal metabolic conditions and risk for autism and other neurodevelopmental disorders Pediatrics 129: e1121-1128. [Crossref]

34. Suren P, Gunnes N, Roth C, Bresnahan M, Hornig M, et al. (2014) Parental obesity and risk of autism spectrum disorder. Pediatrics 133: e1128-1138. [Crossref]

35. Bochukova EG, Huang N, Keogh J, Henning E, Purmann C, et al. (2010) Large, rare chromosomal deletions associated with severe early-onset obesity. Nature 463: 666670. [Crossref]

36. Walters RG, Jacquemont S, Valsesia A, de Smith AJ, Martinet D, et al. (2010) A new highly penetrant form of obesity due to deletions on chromosome 16p11.2. Nature 463: 671-675. [Crossref]

37. Soubry A, Murphy SK, Wang F, Huang Z, Vidal AC, et al. (2015) Newborns of obese parents have altered DNA methylation patterns at imprinted genes. Int $J$ Obes (Lond) 39: 650-657. [Crossref]

38. Yau PL, Castro MG, Tergani A, Tsui WH, Convit A (2012) Obesity and metabolic syndrome and functional and structural brain impairment in adolescence. Pediatrics 130: e856-e65.

39. Gold SM, Dziobek I, Sweat V, Tirsi A, Rogers K et al. Hippocampal damage and memory impairments as possible early brain complications of type 2 diabetes. Diabetologia 50: 711-719.

40. Vargas DL, Nascimbene C, Krishnan C, Zimmerman AW, Pardo CA (2005) Neuroglia activation and neuroinflammation in the brain of patients with autism. Ann Neurol 57: 67-81. [Crossref]

41. Moon RY (2016) AAP Task Force on Sudden Infant Death Syndrome. SIDS and other sleep-related infant deaths: Evidence base for 2016 updated recommendations for a safe infant sleeping environment. Pediatrics 138: ve20162940.

42. Collett B, Breiger D, King D, Cunningham M, Speltz M (2005) Neurodevelopmenta Implications of "Deformational" Plagiocephaly. J Develop Behav Pediat 26: 379-389.

43. Leonard HC, Elsabbagh M, Hill EL \& the BASIS team (2014) Early and persistent motor difficulties in infants at-risk of developing autism spectrum disorder: A prospective study. Eur J Develop Psychol 11: 18-35.

44. Van Waelvelde H, Oostra A, Dewitte G, Van Den Broeck C, Jongmans MJ (2010) Stability of motor problems in young with or at risk of autism spectrum disorders, ADHD, and or developmental coordination disorder. Develop Med Child Neurol 52: e174-e178.

45. Lin T-Y, Lin P-Y, Su T-P, Chen Y-S, Hsu J-W et al. (2014) Autistic spectrum disorder, attention deficit hyperactivity disorder, and allergy: Is there a link? A nationwide study. Res Autism Spect Disord 8: 1333-1338.

46. Ayral M, Baylan MY, Kinis V, Bez Y, Bakir S, Ozbay M et al. (2013) Evaluation of hyperactivity, attention deficit, and impulsivity before and after adenoidectomy/ adenotonsillectomy surgery. J Craniofacial Surg 24: 731-773.

47. Biederman J, Newcorn J, Sprich S (1991) Comorbidity of attention deficit hyperactivity disorder with conduct, depressive, anxiety, and other disorders. Am J Psychol 148: 564577

Copyright: (C2019 Gurevitz M. This is an open-access article distributed under the terms of the Creative Commons Attribution License, which permits unrestricted use, distribution, and reproduction in any medium, provided the original author and source are credited. 